\title{
Scaling law of distributed estimation in sensor networks with semi-orthogonal MAC
}

\author{
Jian Su${ }^{1}$, Ha H Nguyen ${ }^{1 a)}$, and Hoang D. Tuan ${ }^{2}$ \\ ${ }^{1}$ Department of ECE, University of Saskatchewan, Saskatoon, Canada S7N 5 A9 \\ ${ }^{2}$ Engr. and Infor. Tech., University of Technology, Sydney, Australia, NSW 2007
}

a)ha.nguyen@usask.ca

\begin{abstract}
This letter is concerned with distributed estimation in a Gaussian sensor network operating under a semi-orthogonal multiple-access channel (MAC). In such a semi-orthogonal MAC, all sensors are divided into groups and signals from the sensors in each group are directly combined (opposed to be coherently combined) and then transmitted on one orthogonal channel to a fusion center (FC). The behavior of the average distortion in terms of the number of sensors, namely the scaling law, is analyzed. The analytical derivation and simulation results show that the semi-orthogonal MAC with adaptive sensor grouping can achieve the optimal scaling law while requiring only a small amount of feedback from the FC to the sensors.
\end{abstract}

Keywords: sensor networks, distributed estimation, multiple-access channel, scaling law

Classification: Network

\section{References}

[1] M. Gastpar and M. Vetterli, "Power, spatio-temporal bandwidth, and distortion in large sensor networks," IEEE J. Sel. Areas Commun., vol. 23, pp. 745-754, Apr. 2005. DOI:10.1109/JSAC.2005.843542

[2] M. Gastpar, "Uncoded transmission is exactly optimal for a simple Gaussian sensor network," IEEE Trans. Inf. Theory, vol. 54, pp. 5247-5251, Nov. 2008. DOI:10.1109/TIT.2008.929967

[3] J. J. Xiao, S. Cui, Z. Q. Luo, and A. Goldsmith, "Linear coherent decentralized estimation," IEEE Trans. Signal Process., vol. 56, pp. 757-770, Feb. 2008. DOI:10.1109/TSP.2007.906762

[4] S. Cui, J. J. Xiao, Z. Q. Luo, A. Goldsmith, and H. V. Poor, "Estimation diversity and energy efficiency in distributed sensing," IEEE Trans. Signal Process., vol. 55, pp. 4683-4695, Sep. 2007. DOI:10.1109/TSP.2007.896019

[5] J. Su and H. H. Nguyen, "Sensor grouping for linear distributed estimation in a wireless sensor network," Proc. IEEE Inter. Conf. Commun., Jun. 2014. DOI: 10.1109/ICC.2014.6883364

[6] S. M. Kay, Fundamentals of Statistical Signal Processing: Estimation Theory, Prentice-Hall, Inc., Englewood Cliffs, New Jersey, 07632, 1993. 


\section{Introduction}

This letter considers the problem of distributed estimation in a Gaussian sensor network in which a scalar Gaussian random variable is observed in a memoryless fashion by $K$ sensors and each observation is subject to white Gaussian noise. The sensors are connected to a fusion center (FC) via wireless channels. Using data collected from the sensors, the FC estimates the underlying source signal to within the smallest distortion possible. The distortion of such distributed estimation is affected by many factors, such as the number of sensors, the total transmit power and the communication bandwidth. Of particular interest in our study is the behavior of the average distortion as a function of the number of sensors, i.e., the scaling law as defined in [1]. In particular, [1, Theorem 1] tells us that the optimal scaling law achieved by analog transmission is $1 / K$.

Regarding the wireless communications between the sensors and the FC, many different types of multiple access channel (MAC) can be used. The coherent MAC $[1,2,3]$ is an example of analog transmission which can achieve the optimal scaling law of $1 / K$. In the coherent MAC, signals from all sensors are transmitted on a single channel. To obtain coherent combination at the FC, the phases of the wireless channel responses need to be compensated at the sensors, which entails a large amount of channel state information (CSI) feedback from the FC to the sensors. In another analog transmission scheme, namely the orthogonal MAC [4], since orthogonal channels are used by different sensors, no channel phase compensation is required at the sensors. However, reference [4] shows that the orthogonal MAC does not achieve the optimal scaling law.

Recently, a novel analog transmission scheme, known as the semi-orthogonal $M A C$ with sensor grouping, is studied in [5]. Such a scheme offers a better tradeoff among the bandwidth, feedback overhead, and estimation performance than both the coherent and orthogonal MACs. As a further development of [5], this letter establishes the important scaling law achieved by the semi-orthogonal scheme with both fixed and adaptive sensor groupings. In particular, it is proved that the semiorthogonal MAC with adaptive sensor grouping can achieve the optimal scaling law of $1 / K$, even with less feedback overhead as compared to the coherent MAC.

\section{System model}

In a distributed Gaussian sensor network proposed in [5], $K$ sensors are used to monitor a source signal $s$ and communicate their observations to a FC over $N$ orthogonal channels. All sensors are divided into disjoint groups and the sensors within each group are allocated to transmit on one orthogonal channel. Since the consumed bandwidth is proportional to the number of the orthogonal channels, $N$ shall be chosen to be much smaller than $K$ to conserve bandwidth.

The observation of the $i$ th sensor can be expressed as $x_{i}=s+v_{i}, i=$ $1,2, \ldots, K$, where the source signal $s$ and observation noise $v_{i}$ are modeled as Gaussian random variables with zero mean and variances $\sigma_{s}^{2}$ and $\sigma_{v}^{2}$, respectively. The observation signal-to-noise ratio (SNR) is defined as $\gamma_{\mathrm{o}}=\sigma_{s}^{2} / \sigma_{v}^{2}$.

With analog transmission, the $i$ th sensor simply amplifies $x_{i}$ with a gain $a_{i}$ and transmits the result to the $\mathrm{FC}$ on one orthogonal channel. Let $h_{i}, i=1, \ldots, K$, 
represent the channel response from sensor $i$ to the FC. These channel responses are modeled as i.i.d. complex Gaussian random variables with zero mean and unit variance. At the FC, the received signal on the $n$th orthogonal channel is $y_{n}=$ $\left[\sum_{i \in \Omega_{n}} a_{i}\left(s+v_{i}\right) h_{i}\right]+\omega_{n}, n=1,2, \ldots, N$, where $\Omega_{n}$ is the index set of the sensors that transmit on the $n$th orthogonal channel, and $\omega_{n}$ 's are the i.i.d. (over $n$ ) complex AWGN components with zero mean and variance $\sigma_{\omega}^{2}$.

The received signal can be rewritten as $y_{n}=\hat{h}_{n} s+\hat{v}_{n}+\omega_{n}$, where $\hat{h}_{n}=$ $\sum_{i \in \Omega_{n}} a_{i} h_{i}$ and $\hat{v}_{n}=\sum_{i \in \Omega_{n}} a_{i} v_{i} h_{i}$. Since $y_{n}$ is complex, while $s$ is real, the phase of $\hat{h}_{n}$ is compensated to obtain ${ }^{1} \bar{y}_{n}=\mathcal{R}\left\{\hat{h}_{n}^{*} y_{n} /\left|\hat{h}_{n}\right|\right\}=\bar{h}_{n} s+\bar{v}_{n}+\bar{\omega}_{n}$, where $\bar{h}_{n}=\left|\sum_{i \in \Omega_{n}} a_{i} h_{i}\right|, \bar{\omega}_{n}=\mathcal{R}\left\{\hat{h}_{n}^{*} \omega_{n} /\left|\hat{h}_{n}\right|\right\}$ and $\bar{v}_{n}=\mathcal{R}\left\{\hat{h}_{n}^{*}\left(\sum_{i \in \Omega_{n}} a_{i} v_{i} h_{i}\right) /\left|\hat{h}_{n}\right|\right\}$. The above phase compensation discards halves of observation noise and channel noise. Since it is performed at the FC, no phase information is needed at the sensors and feedback of CSI from the FC to the sensors is not required.

Let $\overline{\mathbf{y}}=\left[\bar{y}_{1}, \bar{y}_{2}, \ldots, \bar{y}_{N}\right], \overline{\mathbf{h}}=\left[\bar{h}_{1}, \bar{h}_{2}, \ldots, \bar{h}_{N}\right], \overline{\mathbf{v}}=\left[\bar{v}_{1}, \bar{v}_{2}, \ldots, \bar{v}_{N}\right]$ and $\overline{\boldsymbol{\omega}}=$ $\left[\bar{\omega}_{1}, \bar{\omega}_{2}, \ldots, \bar{\omega}_{N}\right]$. Then one has $\overline{\mathbf{y}}=\overline{\mathbf{h}} s+\overline{\mathbf{v}}+\overline{\boldsymbol{\omega}}$. The LMMSE estimator is adopted at the FC. Accordingly, the estimate of $s$ based on $\overline{\mathbf{y}}$ is [6]

where ${ }^{2}$

$$
\check{s}=\sigma_{s}^{2} \overline{\mathbf{h}}^{\top}\left(\sigma_{s}^{2} \overline{\mathbf{h}}^{\top} \overline{\mathbf{h}}^{\top}+\Sigma_{\overline{\mathbf{v}}}+\Sigma_{\bar{\omega}}\right)^{-1} \overline{\mathbf{y}},
$$

$$
\begin{gathered}
\boldsymbol{\Sigma}_{\overline{\mathbf{v}}}=\mathcal{E}\left\{\overline{\mathbf{v}} \overline{\mathbf{v}}^{\top}\right\}=\operatorname{diag}\left(\sum_{i \in \Omega_{n}} a_{i}^{2} \sigma_{v}^{2} r_{n i}^{2} ; \quad n=1, \ldots, N\right), \\
r_{n i}=\left(\mathcal{R}\left\{h_{i}\right\} \mathcal{R}\left\{\hat{h}_{n}\right\}+\mathcal{I}\left\{h_{i}\right\} \mathcal{I}\left\{\hat{h}_{n}\right\}\right) /\left|\hat{h}_{n}\right|, \\
\boldsymbol{\Sigma}_{\bar{\omega}}=\mathcal{E}\left\{\overline{\boldsymbol{\omega}} \overline{\boldsymbol{\omega}}^{\top}\right\}=\operatorname{diag}\left(\frac{\sigma_{\omega}^{2}}{2}, \frac{\sigma_{\omega}^{2}}{2}, \ldots, \frac{\sigma_{\omega}^{2}}{2}\right) .
\end{gathered}
$$

The corresponding MSE distortion is

$$
\left[\sigma_{s}^{-2}+\overline{\mathbf{h}}^{\top}\left(\boldsymbol{\Sigma}_{\overline{\mathbf{v}}}+\boldsymbol{\Sigma}_{\bar{\omega}}\right)^{-1} \overline{\mathbf{h}}\right]^{-1}=\left[\sigma_{s}^{-2}+\sum_{n=1}^{N} \frac{\left|\sum_{i \in \Omega_{n}} a_{i} h_{i}\right|^{2}}{\left(\sum_{i \in \Omega_{n}} a_{i}^{2} r_{n i}^{2}\right) \sigma_{v}^{2}+\frac{\sigma_{\omega}^{2}}{2}}\right]^{-1} .
$$

\section{Performance analysis}

First, consider equal power allocation, i.e., $a_{i}=\bar{a}=\sqrt{P_{\text {tot }} /\left[K\left(\sigma_{s}^{2}+\sigma_{v}^{2}\right)\right]}$. This simplifies (5) to

$$
\epsilon=\sigma_{s}^{2}\left[1+\sum_{n=1}^{N} \frac{\left|\sum_{i \in \Omega_{n}} \frac{h_{i}}{\sqrt{K}}\right|^{2}}{\left(\sum_{i \in \Omega_{n}} \frac{r_{n i}^{2}}{K}\right) \frac{1}{\gamma_{0}}+\frac{1}{2 \gamma_{\mathrm{c}}}\left(1+\frac{1}{\gamma_{0}}\right)}\right]^{-1},
$$

where $\gamma_{\mathrm{c}}=P_{\text {tot }} / \sigma_{\omega}^{2}$ is the channel SNR. In the following, for simplicity, assume $\sigma_{s}^{2}=1$ and define $\gamma_{c}^{\prime}=\gamma_{c} /\left(1+\frac{1}{\gamma_{0}}\right)$.

The main objective of this letter is to establish the scaling law achieved by the semi-orthogonal MAC. The scaling law, as defined in [1], is the decaying rate that the average MSE (AMSE) distortion achieves as $K$ increases. In the following, the AMSE of different cases of semi-orthogonal MAC is examined in more detail.

\subsection{Semi-orthogonal MAC with fixed sensor grouping}

With sensor grouping, $N$ is fixed to a small value to save bandwidth. For fixed sensor grouping (FSG), $K$ sensors are divided into $N$ disjoint groups such that

\footnotetext{
${ }^{1}$ For complex quantities, $\mathcal{R}\{\cdot\}$ denotes the real part and $\mathcal{I}\{\cdot\}$ denotes the imaginary part.

${ }^{2}$ For random variables, $\mathcal{E}\{\cdot\}$ denotes expectation
} 
$\Omega_{n}=\{(n-1)(K / N)+1, \ldots, n(K / N)]$ and the sensors in each group transmit on one orthogonal channel. In this case, $K$ is an integer multiple of $N$. The strategy of FSG means that the orthogonal channel used by each sensor, once assigned, does not change during the communication phase. As a result, no feedback of channel information is required in this scheme. Based on (6), a lower bound on the AMSE of the semi-orthogonal MAC with FSG can be found as

$$
\mathcal{E}\{\epsilon\} \geq \mathcal{E}\left\{\left(1+\sum_{n=1}^{N} \frac{\left|\sum_{i \in \Omega_{n}} \frac{h_{i}}{\sqrt{K}}\right|^{2}}{\frac{1}{2 \gamma_{\mathrm{c}}^{\prime}}}\right)^{-1}\right\} \geq\left(1+\sum_{n=1}^{N} \frac{\mathcal{E}\left\{\left|\sum_{i \in \Omega_{n}} \frac{h_{i}}{\sqrt{K}}\right|^{2}\right\}}{\frac{1}{2 \gamma_{\mathrm{c}}^{\prime}}}\right)^{-1}
$$

Since $\sum_{i \in \Omega_{n}} h_{i} / \sqrt{K}$ is a complex Gaussian random variable with zero mean and variance $1 / N$, the lower bound turns to

$$
\mathcal{E}\{\epsilon\} \geq\left(1+\sum_{n=1}^{N} \frac{\frac{1}{N}}{\frac{1}{2 \gamma_{\mathrm{c}}^{\prime}}}\right)^{-1}=\left(1+2 \gamma_{\mathrm{c}}^{\prime}\right)^{-1} .
$$

The above lower bound is independent of $K$, which means that the optimal scaling law of $1 / K$ cannot be achieved by the semi-orthogonal MAC with FSG.

\subsection{Semi-orthogonal MAC with adaptive sensor grouping}

For the adaptive sensor grouping (ASG), the sensors are grouped based on the phases of their channel responses. To this end, the whole phase region of $2 \pi$ are partitioned into $N$ equal sub-regions (each of length $2 \pi / N$ ), and the sensors with channel phases in the same sub-region are assigned to transmit on the same orthogonal channel. In this case, feedback of orthogonal channel allocation from the FC to the sensors is required. For each sensor, $\log _{2} N$ bits are needed, which is a much smaller amount of feedback compared to the CSI required in the coherent MAC, especially when $N$ is small. The scaling law achieved by the semi-orthogonal MAC with ASG is stated in the following theorem.

Theorem 1. The AMSE achieved by the semi-orthogonal MAC with ASG scales like $1 / K$ when $K \rightarrow \infty$, i.e., $\lim _{K \rightarrow \infty} K \mathcal{E}\{\epsilon\}=c$, for some constant $c>0$.

Proof. First, Equation (6) is rewritten in the following form:

$$
\epsilon=\left\{1+\sum_{n=1}^{N} \frac{\frac{K_{n}^{2}}{K}\left[\left(\sum_{i \in \Omega_{n}} \frac{x_{i}}{K_{n}}\right)^{2}+\left(\sum_{i \in \Omega_{n}} \frac{y_{i}}{K_{n}}\right)^{2}\right]}{\frac{K_{n}}{K}\left(\sum_{i \in \Omega_{n}} \frac{r_{n i}^{2}}{K_{n}}\right) \frac{1}{\gamma_{o}}+\frac{1}{2 \gamma_{c}^{\prime}}}\right\}^{-1},
$$

where $x_{i}$ and $y_{i}$ are, respectively, the real and imaginary parts of $h_{i}$, and $K_{n}$ is the size of $\Omega_{n}$. When $K \rightarrow \infty, K_{n}$ approaches to its mean value $K / N$ (which is also infinity). Then it follows from the strong law of large numbers that when $K_{n} \rightarrow \infty$ one has $\sum_{i \in \Omega_{n}} x_{i} / K_{n} \stackrel{\text { a.e. }}{\longrightarrow} \mathcal{E}\left\{x_{i}\right\}, \sum_{i \in \Omega_{n}} y_{i} / K_{n} \stackrel{\text { a.e. }}{\longrightarrow} \mathcal{E}\left\{y_{i}\right\}$ and $\sum_{i \in \Omega_{n}} r_{n i}^{2} / K_{n} \stackrel{\text { a.e. }}{\longrightarrow}$ $\mathcal{E}\left\{r_{n i}^{2}\right\}$. It then follows that

$$
\epsilon \stackrel{\text { a.e. }}{\longrightarrow}\left[1+\sum_{n=1}^{N} \frac{\frac{K}{N^{2}}\left(\mathcal{E}^{2}\left\{x_{i}\right\}+\mathcal{E}^{2}\left\{y_{i}\right\}\right)}{\frac{1}{N} \mathcal{E}\left\{r_{n i}^{2}\right\} \frac{1}{\gamma_{0}}+\frac{1}{2 \gamma_{\mathrm{c}}^{\prime}}}\right]^{-1} .
$$

As $K \rightarrow \infty$, all sensor groups have identical distributions of channel responses. 


$$
\epsilon \stackrel{\text { a.e. }}{\longrightarrow}\left[1+\frac{N \frac{K}{N^{2}}\left(\mathcal{E}^{2}\left\{x_{i}\right\}+\mathcal{E}^{2}\left\{y_{i}\right\}\right)}{\frac{1}{N} \mathcal{E}\left\{r_{n i}^{2}\right\} \frac{1}{\gamma_{\mathrm{o}}}+\frac{1}{2 \gamma_{c}^{\prime}}}\right]^{-1}=\left[1+K \frac{\left(\mathcal{E}^{2}\left\{x_{i}\right\}+\mathcal{E}^{2}\left\{y_{i}\right\}\right)}{\mathcal{E}\left\{r_{n i}^{2}\right\} \frac{1}{\gamma_{\mathrm{o}}}+\frac{N}{2 \gamma_{c}^{\prime}}}\right]^{-1} .
$$

For the ASG, the channel responses in sensor group $n$ have i.i.d. distribution of

$$
\frac{N}{\pi} \exp \left[-\left(x_{i}^{2}+y_{i}^{2}\right)\right], \quad \frac{2(n-1) \pi}{N} \leq \theta_{i}<\frac{2 n \pi}{N},
$$

where $\theta_{i}$ is the phase of $h_{i}$. Based on this distribution function, one has $\mathcal{E}\left\{x_{i}\right\}=(N / 2 \sqrt{\pi}) \cos \alpha \sin \beta, \quad \mathcal{E}\left\{y_{i}\right\}=(N / 2 \sqrt{\pi}) \sin \alpha \sin \beta, \quad \mathcal{E}\left\{x_{i}^{2}\right\}=$ $(N / 4 \pi) \cos 2 \alpha \sin 2 \beta+1 / 2, \mathcal{E}\left\{y_{i}^{2}\right\}=-(N / 4 \pi) \cos 2 \alpha \sin 2 \beta+1 / 2$ and $\mathcal{E}\left\{x_{i} y_{i}\right\}=$ $(N / 4 \pi) \sin 2 \alpha \sin 2 \beta$, where $\alpha=(2 n-1) \pi / N$ and $\beta=\pi / N$. In addition,

$$
\begin{aligned}
\mathcal{E}\left\{r_{n i}^{2}\right\} & =\mathcal{E}\left\{\left(x_{i} \cos \varphi_{n}+y_{i} \sin \varphi_{n}\right)^{2}\right\} \\
& =\mathcal{E}\left\{x_{i}^{2}\right\} \cos ^{2} \varphi_{n}+\mathcal{E}\left\{y_{i}^{2}\right\} \sin ^{2} \varphi_{n}+2 \mathcal{E}\left\{x_{i} y_{i}\right\} \cos \varphi_{n} \sin \varphi_{n},
\end{aligned}
$$

where $\varphi_{n}$ is the phase of $\hat{h}_{n}$.

It is easy to prove that when $K \rightarrow \infty, \varphi_{n}$ approaches $\mathcal{E}\left\{\theta_{i}\right\}=(2 n-1) \pi / N$ with probability 1 . Thus, $\mathcal{E}\left\{r_{n i}^{2}\right\} \stackrel{\text { a.e. }}{\longrightarrow} \frac{1}{2}+\frac{N}{4 \pi} \sin \left(\frac{2 \pi}{N}\right)$. Therefore, when $K \rightarrow \infty$,

$$
\epsilon \stackrel{\text { a.e. }}{\longrightarrow}\left\{1+K \frac{\frac{N^{2}}{4 \pi} \sin ^{2}\left(\frac{\pi}{N}\right)}{\left[\frac{1}{2}+\frac{N}{4 \pi} \sin \left(\frac{2 \pi}{N}\right)\right] \frac{1}{\gamma_{o}}+\frac{N}{2 \gamma_{c}^{\prime}}}\right\}^{-1} \text {. }
$$

Finally,

$$
\lim _{K \rightarrow \infty} K \mathcal{E}\{\epsilon\}=\frac{\left[\frac{1}{2}+\frac{N}{4 \pi} \sin \left(\frac{2 \pi}{N}\right)\right] \frac{1}{\gamma_{o}}+\frac{N}{2 \gamma_{c}^{\prime}}}{\frac{N^{2}}{4 \pi} \sin ^{2}\left(\frac{\pi}{N}\right)},
$$

which is a constant.

The analysis of the scaling laws in this section is valid for equal power allocation. If the optimal power allocation ${ }^{3}$ can be found, whether the optimal scaling law of $1 / K$ can be achieved by the semi-orthogonal MAC with FSG remains to be answered and deserves a further study. On the other hand, for the semi-orthogonal MAC with ASG, the optimal scaling law has already been achieved with the equal power allocation and surely it is also achieved with the optimal power allocation.

\section{Simulation results}

In all simulations, it is assumed that $\gamma_{\mathrm{o}}=20 \mathrm{~dB}$ and $\gamma_{\mathrm{c}}=25 \mathrm{~dB}$. Fig. 1 plots the AMSEs of the orthogonal MAC and the semi-orthogonal MAC with FSG versus the number of sensors, $K$. As $K$ increases, the AMSEs of both MACs asymptotically converge to positive constants. For the semi-orthogonal MAC with FSG, the lower bound of $\left(1+2 \gamma_{\mathrm{c}}^{\prime}\right)^{-1}$ obtained in (8) is quite loose for small values of $K$ and $N$, but it becomes tighter when $K$ and $N$ get larger.

The scaling law achieved by the semi-orthogonal MAC with ASG is illustrated in Fig. 2, where the AMSEs of the orthogonal and coherent MACs are also provided for comparison. Similar to the coherent MAC, the AMSE of the semiorthogonal MAC with ASG appears as a straight line when $K$ is large. Since the plots are in log-log fashion, a straight line means that the AMSE decays in an order of $1 / K$ as $K$ increases, showing that the optimal scaling law of the studied analog

\footnotetext{
${ }^{3}$ The optimal power allocation divides the total power among sensors to obtain the smallest instantaneous MSE. Such a technique requires extra feedback from the FC to sensors.
} 


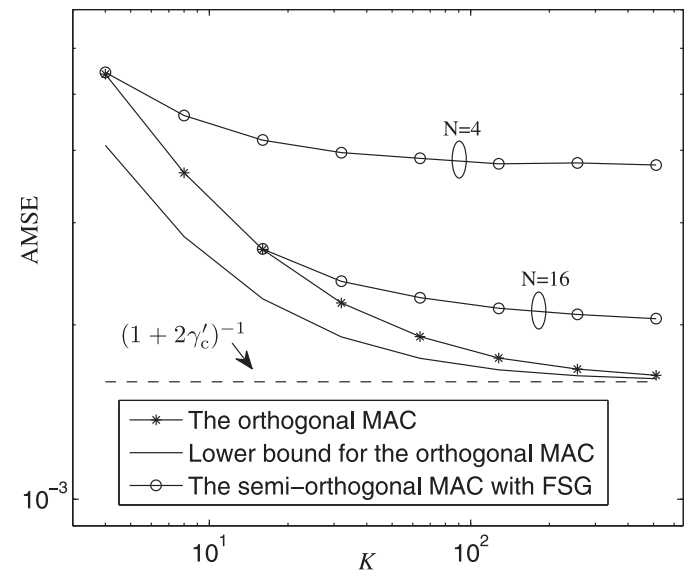

Fig. 1. AMSE and its lower bound for the orthogonal MAC and the semi-orthogonal MAC with FSG.

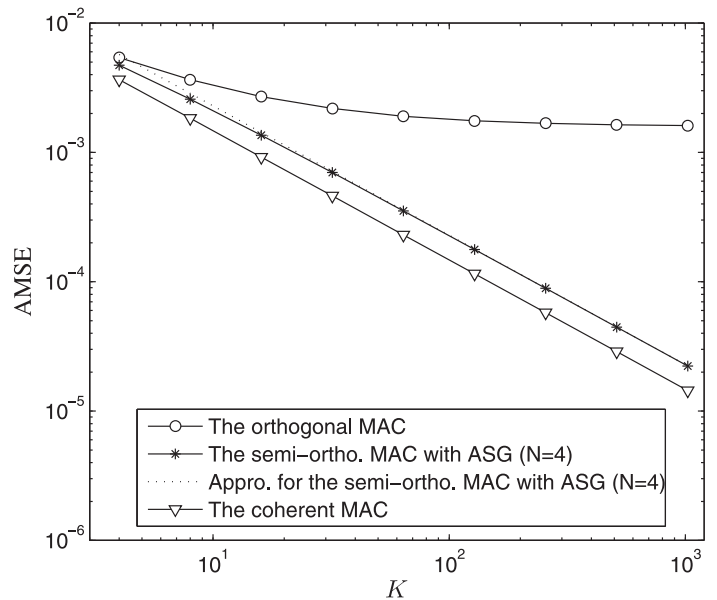

Fig. 2. AMSE and its approximation for the semi-orthogonal MAC with ASG.

Gaussian sensor networks is achieved. In addition, it can be shown that the constant $c$ of the ASG scheme (Eq. (15)) is larger than that of the coherent MAC. This means that the ASG scheme has a higher distortion compared to the coherent MAC with the same number of sensors. Therefore, while the semi-orthogonal MAC with ASG is as optimal as the coherent MAC in the scaling-law sense, it requires more sensors than the coherent MAC to achieve the same distortion.

\section{Conclusions}

The AMSE distortions of distributed estimation under two versions of the semiorthogonal MAC, namely fixed sensor grouping and adaptive sensor grouping, are analyzed under equal power allocation. The semi-orthogonal MAC with FSG requires no feedback from the $\mathrm{FC}$ to sensors but fails to achieve the optimal scaling law. In contrast, for the semi-orthogonal MAC with ASG, the optimal scaling law of $1 / K$ can be achieved. This means that the estimation distortion can be decreased to an arbitrary low level by employing more sensors. Compared to the coherent MAC, such optimal scaling law of the semi-orthogonal MAC with ASG can be achieved with a much smaller amount of feedback. 